\title{
COMPARISON OF MEAN LIGHT CURVE PARAMETERS OF $M, S$ AND C MIRA AND SEMI-REGULAR VARIABLE STARS USING 75 YEARS OF AAVSO DATA
}

\author{
MARIE-ODILE MENNESSIER AND HICHAME BOUGHALEB \\ GRAAL, Université de Montpellier II \\ F-34095 Montpellier, France \\ AND \\ JANET A. MATTEI \\ American Association of Variable Star Observers \\ Cambridge, MA 02138, U.S.A.
}

\begin{abstract}
Using 75 years of AAVSO data, mean light curve parameters of a sample of 350 long-period M, S and C Mira and semi-regular variable stars have been investigated. We compare M, S and C Mira and semiregular stars, present a classification of the light curves of LPVs and give discriminant parameters.
\end{abstract}

\section{Introduction}

From 75 years of AAVSO data - dates and magnitudes of maxima and minima - of M, S and C Mira and semi-regular variable stars used during the preparation of the HIPPARCOS mission, we have performed a cluster analysis of the mean light curves and have examined the peculiar characteristics of each cluster.

It is well known that the differentiation between carbon- and oxygenrich Mira stars is not obvious from photometric criteria. We show a way for identifying $\mathrm{C}$ Miras from our light curve classification and IRAS colors.

Another point is that long-period variable stars may show long-term variations. In the last part of this paper we give some indications of these possible long-term variations derived from the light curve parameters. 


\section{Classification of Light Curves}

\subsection{PARAMETERS}

We characterize the mean light curve of each star by:

- The mean period $P$ from maximum to maximum,

- The mean amplitude $A$ of optical brightness between one maximum and the following minimum, and

- The asymmetry $f$, i.e. the mean ratio between the rising time and the period.

The corresponding values can be found in the data base ASTRID (1996).

\subsection{CLASSIFICATION}

Using the above parameters as variables we perform a dynamical clustering (Murtagh \& Heck 1987) that leads to a classification into 6 clusters. The characteristics of the six best clusters according to the combinations found to describe them best are given in Table 1, and the numbers of stars of the different types in each of these clusters are given in Table 2.

TABLE 1. The characteristics of the six best clusters as achieved from an automatic classification (dynamical clustering); $n$ is the number of stars belonging to each cluster.

\begin{tabular}{|c|c|cccc|}
\hline $\begin{array}{c}\text { Best } \\
\text { cluster }\end{array}$ & $n$ & $P$ & $A$ & $100 f-5.9 A$ & $100 f-0.022 P$ \\
\hline C2 & 110 & $\lesssim 275$ & $>3.7$ & $>10.5 \&<26$ & - \\
C4 & 67 & $\gtrsim 275$ & $>3.7$ & $>10.5 \&<26$ & $>35$ \\
C1 & 54 & $\gtrsim 275$ & $<4.7$ & $>10.5 \&<26$ & $<35$ \\
C6 & 51 & $\gtrsim 275$ & $>4.7$ & $<10.5$ & $<35$ \\
C5 & 37 & $\lesssim 275$ & $<3.7$ & $>26$ & - \\
C3 & 35 & $\gtrsim 275$ & $<3.7$ & $>26$ & $>35$ \\
& & & & & \\
\hline
\end{tabular}

\subsection{WHAT ABOUT C STARS?}

We confirm the well-known characteristics:

- There are neither $\mathrm{C}$ stars nor semi-regular variables in the clusters corresponding to large-amplitude light curves, i.e. clusters 2, 4 and 6; 
TABLE 2. Number of stars in each cluster according to different spectral and variability types.

\begin{tabular}{|c|cccccc|}
\hline Type & C1 & C2 & C3 & C4 & C5 & C6 \\
\hline O rich & & & & & & \\
Miras & 48 & 106 & 12 & 59 & 25 & 40 \\
SRs & 1 & & 3 & & 12 & \\
C rich & & & & & & \\
Miras & 4 & & 15 & & 1 & 1 \\
SRs & & & 4 & & & \\
S & 1 & 4 & 1 & 8 & & 10 \\
\hline
\end{tabular}

- The majority (80\%) of C-rich variable stars and some (5\%) O-rich Mira stars have a long periods, small amplitudes, and symmetric light curves, i.e. they belong to the same cluster 3 .

We thus conclude that:

- The light curves of carbon stars appear to be in the continuum of semi-regular variables with longer period in $(P, A, f)$ space;

- The taxonomy from mean light curve parameters is not sufficient to differentiate C-rich from O-rich variables.

\section{Discrimination of C Stars}

The $\mathrm{M}$ or $\mathrm{C}$ spectral types for long-period variable stars depend on the abundance ratio $\mathrm{C} / \mathrm{O}$ in the surface layers of these stars. Although some dust features are observed in both types, it is well known that IRAS colors alone are not discriminant parameters for C stars. Epchtein et al. (1987) propose to use the $K$ and $L$ photometric fluxes in conjunction with IRAS data. If different $\mathrm{C} / \mathrm{O}$ ratios induce different properties in the circumstellar medium, we propose that the combination of light curve classification and IRAS colors may reflect the stellar and circumstellar properties and thus could distinguish carbon-rich from oxygen-rich stars.

We consider only IRAS fluxes of quality 3 , and we use the ([12] - [25], $[25]-[60]$ ) color-color diagram. We draw the following conclusions (Mennessier et al. 1997): 
- IRAS colors of the oxygen-rich long-period variables belonging to cluster 3 are different from those of $\mathrm{C}$ stars belonging to this cluster;

- the oxygen-rich long-period variables with the same IRAS colors as the C stars belong to different clusters.

The same properties are true also when we extend the sample to all Mira stars included in the General Catalogue of Variable Stars with IRAS fluxes of quality 3 (Mennessier et al. 1997).

Thus the combination of light curve classification and IRAS colors allows the discrimination of carbon Miras.

\section{Long Time Variations}

We know that long-period variables exhibit variations from one cycle to another and can even present systematic long-term trends in period and amplitude (Mattei \& Foster 2000). A way to detect such variations is to compute the correlation coefficients of the variables defined in $\S 2.1$, two by two, between consecutive cycles (Boughaleb 1994). One of the most interesting significant coefficients is a positive one between magnitudes at successive maxima, i.e. a tendency for successive bright (faint) maxima. Table 3 gives the number of stars with such a significant correlation by type of variability and cluster.

TABLE 3. Number of stars with a light curve having a significant positive correlation coefficient between consecutive cycles in each cluster according to different types.

\begin{tabular}{|c|cccccc|c|}
\hline Type & C1 & C2 & C3 & C4 & C5 & C6 & \\
\hline O rich & & & & & & \\
Miras & 3 & 1 & 7 & 4 & 7 & & $22 / 290$ \\
SRs & 1 & & 1 & & 5 & & $7 / 16$ \\
C rich & & & & & & \\
Miras & 1 & & 13 & & & $14 / 21$ \\
SRs & & & 4 & & & & $4 / 4$ \\
S & 1 & 1 & & 2 & & 2 & $6 / 24$ \\
\hline
\end{tabular}


It can be seen that:

- The correlation is mainly found for stars belonging to the clusters 3 (about $50 \%$ for $\mathrm{M}$ stars and $90 \%$ for $\mathrm{C}$ stars) and 5 (30\%), i.e. for small-amplitude, symmetric light curves with any periods.

- The number of light curves with this correlation is much higher for C-rich (75\%) than for O-rich stars (less than 10\%). It could be intermediate for S stars (about 25\%) but we urge caution due to the small number of stars.

This agrees with a long time variation and is consistent with the models developed by Winters et al. (2000) at Berlin.

\section{Conclusion}

The main results of our light curve studies of long-period variable stars are:

- Mean parameters of the visual light curves can be used with IRAS colors to discriminate between oxygen-rich and carbon-rich Mira stars;

- There is a similarity of some light curve parameters of C Miras and semi-regular variables;

- A tendency for a long time variability is detected in most C Miras and all C semi-regular variables. This could be linked with the influence of dust on the visual light curves and probably depends on the carbon abundance.

\section{References}

ASTRID: "Advanced Stars: a Tool for Relating Information and Data," 1996, http://graal.univ-montp2.fr

Boughaleb, H. 1994, Ph.D. dissertation, Univ. Montpellier II

Epchtein, N., Le Bertre, T., Lépine, J. R. D., Marques dos Santos, P., Matsuura, O. T. \& Picazzio, E. 1987, A\&A Supp., 71, 39

Mattei, J. A. \& Foster, G. 2000, in IAU Symp. 177: The Carbon Star Phenomenon, ed. R. F. Wing (Kluwer), p. 155

Mennessier, M. O., Boughaleb, H. \& Mattei, J. A. 1997, A\&A Supp., 124, 143

Murtagh, F. \& Heck, A. 1987, Multivariate Data Analysis, D. Reidel Publ. Co.

Winters, J. M., Fleischer, A. J., Le Bertre, T. \& Sedlmayr, E. 2000, in IAU Symp. 177: The Carbon Star Phenomenon, ed. R. F. Wing (Kluwer), p. 590

\section{Discussion}

Little-Marenin: Could your method be used to estimate the spectral classes of stars in the AAVSO database with unknown spectral classes?

Mennessier: Yes. 


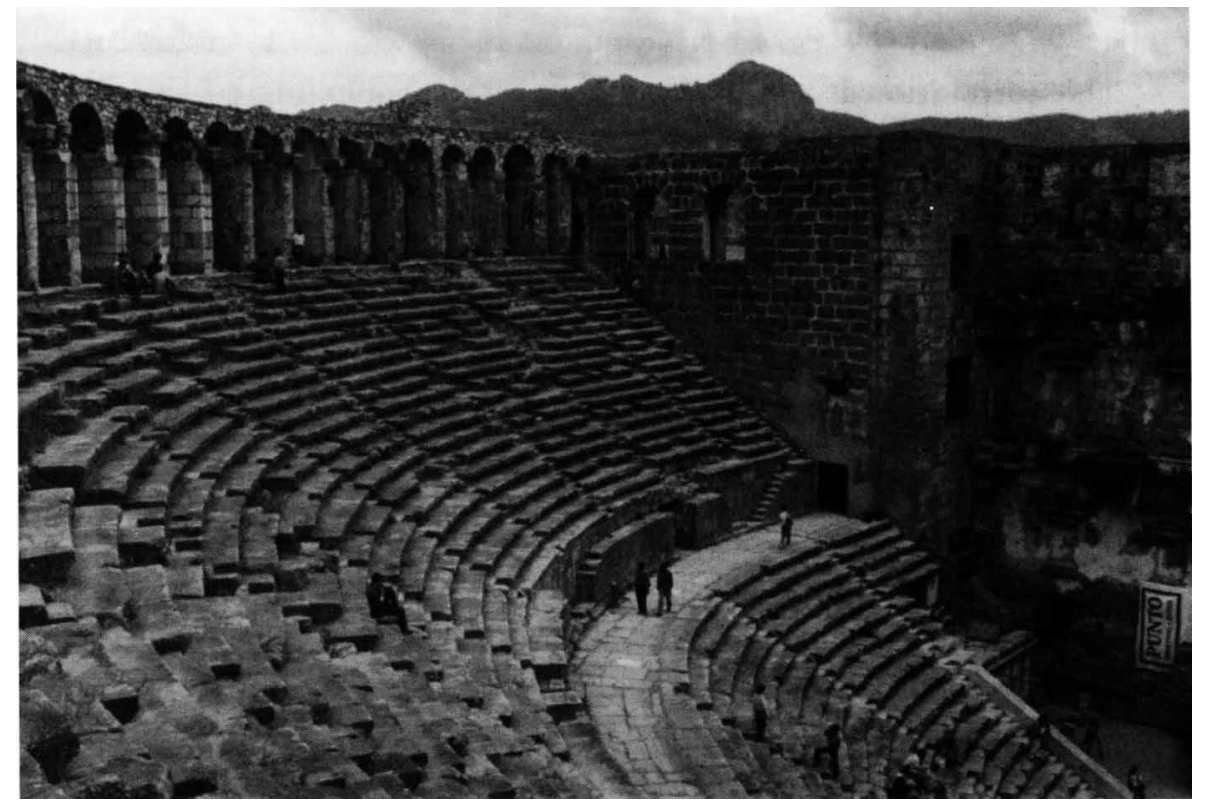

The great theater at Aspendos, dating from 150 A.D., is said to be the best-preserved theater of the classical world. 